\title{
Classical limit in terms of symbolic dynamics for the quantum baker's map
}

\author{
Andrei N. Soklakov* and Rüdiger Schack ${ }^{\dagger}$ \\ Department of Mathematics, Royal Holloway, University of London, \\ Egham, Surrey TW20 0EX, UK \\ and \\ Center for Advanced Studies, Department of Physics and Astronomy, \\ University of New Mexico, Albuquerque, New Mexico 87131-1156, USA.
}

12 August 1999

\begin{abstract}
We derive a simple closed form for the matrix elements of the quantum baker's map that shows that the map is an approximate shift in a symbolic representation based on discrete phase space. We use this result to give a formal proof that the quantum baker's map approaches a classical Bernoulli shift in the limit of a small effective Planck's constant.
\end{abstract}

\section{Introduction}

The quantum baker's map [1], 2] is a prototypical quantum map invented for the theoretical study of quantum chaos. During the last decade, its semiclassical properties have been studied extensively [3, 4, 5, 6, 7, 8], it has been shown to display hypersensitivity to perturbation [9, 10], optical [11] and quantum computing [12, 13] realizations have been proposed, its long-time behavior has been investigated [4, 7], it has been studied in a path-integral approach [8] and defined on a sphere [14]. The quantum baker's map is a quantized version of the classical baker's transformation [15], but there is no unique quantization procedure [16]. The original definition of the map [1, 2] is based on Weyl's quantization [17] of the unit square. Essentially the same map has been derived by algebraic methods [18, 19] as well as by considering the transition from ray to wave optics [11]. Recently a whole class of quantum baker's maps has been defined [20] by exploiting formal similarities between the symbolic dynamics [21] for the classical map on the one hand and the dynamics of strings of quantum bits of the type considered in the theory of quantum computing on the other hand. This class of quantum baker's maps, which can also be derived from the semiquantum maps introduced in Ref. [5], is the subject of this paper.

*a.soklakov@rhul.ac.uk

† r.schack@rhul.ac.uk 
The classical baker's transformation [15] maps the unit square $0 \leq q, p \leq 1$ onto itself according to

$$
(q, p) \longmapsto \begin{cases}\left(2 q, \frac{1}{2} p\right), & \text { if } 0 \leq q \leq \frac{1}{2} \\ \left(2 q-1, \frac{1}{2}(p+1)\right), & \text { if } \frac{1}{2}<q \leq 1 .\end{cases}
$$

This corresponds to compressing the unit square in the $p$ direction and stretching it in the $q$ direction, while preserving the area, then cutting it vertically, and finally stacking the right part on top of the left part - in analogy to the way a baker kneads dough. The classical baker's map, has a simple description in terms of its symbolic dynamics 21. Each point $(q, p)$ is represented by a symbolic string

$$
s=\cdots s_{-2} s_{-1} s_{0} \cdot s_{1} s_{2} \cdots,
$$

where $s_{k}=0$ or 1 , and

$$
q=\sum_{k=1}^{\infty} s_{k} 2^{-k}, \quad p=\sum_{k=0}^{\infty} s_{-k} 2^{-k-1} .
$$

The action of the baker's map on a symbolic string $s$ is then given by the shift map (or Bernoulli shift) $U$ defined by $U s=s^{\prime}$, where $s_{k}^{\prime}=s_{k+1}$. This means that, at each time step, the entire string is shifted one place to the left while the dot remains fixed. Although the relation (3) between points $(q, p)$ and symbolic strings is particular to the baker's transformation, the method of symbolic dynamics is very general and can be applied to a large class of chaotic maps [21].

Symbolic representations for the quantum baker's map have been introduced in Refs. [5, 12, 20]. These representations are all obtained by writing the quantum propagator $\hat{B}$ in a mixed form $\left\langle i|\hat{B}| i^{\prime}\right\rangle$, where $\{|i\rangle\}$ and $\left\{\left|i^{\prime}\right\rangle\right\}$ are different bases. In this paper, we derive a simple closed form of the matrix elements with respect to a single basis. We show that all members of the class of quantum baker's maps defined in Ref. [20] are approximate shifts in a symbolic representation based on discrete phase space. We use this result to give a formal proof that all members of this class of quantum baker's maps approach a classical Bernoulli shift in the limit of a small effective Planck's constant.

The paper is organized as follows. In Sec. 2, we give the necessary background and definitions. In Sec. 3, we state the results of the paper and discuss their significance. Finally, Sec. t contains the derivations and proofs.

\section{$2 \quad$ Background}

Most results of this paper are phrased in terms of finite binary strings. It will be convenient to adopt a slightly different and more flexible notation than the one used in Eq. (2). Here, a binary string

$$
\xi_{s: f} \stackrel{\text { def }}{=} \begin{cases}\xi_{s} \xi_{s+1} \cdots \xi_{f} & (s \leq f) \\ \xi_{s} \xi_{s-1} \cdots \xi_{f} & (s>f)\end{cases}
$$

where $\xi_{i} \in\{0,1\}$ is a bit, can have increasing $(s<f)$ or decreasing $(s>f)$ indices. We will use bold Greek and Latin letters to denote binary strings, e.g.,

$$
\boldsymbol{\alpha}=\xi_{s: f} \quad \text { or } \boldsymbol{x}=x_{h: t} .
$$


The length of a string $\boldsymbol{\alpha}$ will be denoted by $|\boldsymbol{\alpha}|$; e.g., in the above example, $|\boldsymbol{\alpha}|=|f-s|+1$. Concatenation of strings is defined in the usual way. Again considering the above example, $\boldsymbol{\alpha} \boldsymbol{x}$ is the string $\boldsymbol{\alpha} \boldsymbol{x}=\xi_{s} \ldots \xi_{f} x_{h} \ldots x_{t}$. Any string $\boldsymbol{\alpha}$ represents a natural number through its binary expansion

$$
\boldsymbol{\alpha}=\sum_{k=1}^{|\boldsymbol{\alpha}|} 2^{|\boldsymbol{\alpha}|-k} \boldsymbol{\alpha}_{(k)}
$$

where $\boldsymbol{\alpha}_{(k)}$ denotes the $k$-th bit of $\boldsymbol{\alpha}, 1 \leq k \leq|\boldsymbol{\alpha}|$, such that

$$
\boldsymbol{\alpha}=\boldsymbol{\alpha}_{(1)} \boldsymbol{\alpha}_{(2)} \ldots \boldsymbol{\alpha}_{(|\boldsymbol{\alpha}|)}
$$

Thus our notation does not distinguish between a binary string and the corresponding natural number. Similarly, two strings $\boldsymbol{\alpha}$ and $\boldsymbol{x}$ can be combined to represent a rational number

$$
\boldsymbol{\alpha} . \boldsymbol{x} \stackrel{\text { def }}{=} \sum_{k=1}^{|\boldsymbol{\alpha}|} 2^{|\boldsymbol{\alpha}|-k} \boldsymbol{\alpha}_{(k)}+\sum_{k=1}^{|\boldsymbol{x}|} 2^{-k} \boldsymbol{x}_{(k)} .
$$

Quantum baker's maps are defined on the $D$-dimensional Hilbert space of the quantized unit square [17]. For consistency of units, we let the quantum scale on "phase space" be $2 \pi \hbar=1 / D$. Following Ref. [2], we choose half-integer eigenvalues $q_{j}=\left(j+\frac{1}{2}\right) / D, j=0, \ldots, D-1$, and $p_{k}=\left(k+\frac{1}{2}\right) / D, k=0, \ldots, D-1$, of the discrete "position" and "momentum" operators $\hat{q}$ and $\hat{p}$, respectively, corresponding to antiperiodic boundary conditions. We further assume that $D=2^{N}$, which is the dimension of the Hilbert space of $N$ qubits, i.e., $N$ two-state systems.

The $D=2^{N}$ dimensional Hilbert space modeling the unit square can be realized as the product space of $N$ qubits in such a way that

$$
\left|q_{j}\right\rangle=\left|\xi_{1}\right\rangle \otimes\left|\xi_{2}\right\rangle \otimes \cdots \otimes\left|\xi_{N}\right\rangle
$$

where $j=\sum_{l=1}^{N} \xi_{l} 2^{N-l}, \xi_{l} \in\{0,1\}$, and where each qubit has basis states $|0\rangle$ and $|1\rangle$. It follows that, written in our string notation as binary numbers, $j=\xi_{1} \xi_{2} \ldots \xi_{N}=\xi_{1: N}$ and $q_{j}=0 . \xi_{1} \xi_{2} \ldots \xi_{N} 1=$ $0 . \xi_{1: N} 1$. We define the notation

$$
\left|. \xi_{1: N}\right\rangle=\left|. \xi_{1} \xi_{2} \ldots \xi_{N}\right\rangle=e^{i \pi / 2}\left|q_{j}\right\rangle
$$

which is closely analogous to Eq. (2), where the bits to the right of the dot specify the position variable; see Ref. 20] for the reason for the phase shift $e^{i \pi / 2}$.

Momentum and position eigenstates are related through the quantum Fourier transform operator $\hat{F}$ [2], i.e., $\hat{F}\left|q_{k}\right\rangle=\left|p_{k}\right\rangle$. Again in analogy to Eq. (2), we define the notation $\left|\xi_{1: N} \cdot\right\rangle=\left|p_{k}\right\rangle$, where $p_{k}=0 . \xi_{N: 1} 1$.

By applying a partial quantum Fourier transform [20] to the position eigenstates, one obtains the family of states

$$
\begin{aligned}
\left|\xi_{1: n} . \xi_{n+1: N}\right\rangle \stackrel{\text { def }}{=} & \left|\xi_{n+1}\right\rangle \otimes \cdots \otimes\left|\xi_{N}\right\rangle e^{i \pi\left(0 . \xi_{n: 1} 1\right)} \otimes \\
& \sqrt{1 / 2}\left\{|0\rangle+\exp \left[2 \pi i\left(0 . \xi_{1} 1\right)\right]|1\rangle\right\} \otimes \\
& \sqrt{1 / 2}\left\{|0\rangle+\exp \left[2 \pi i\left(0 . \xi_{2} \xi_{1} 1\right)\right]|1\rangle\right\} \otimes \cdots \otimes \\
& \sqrt{1 / 2}\left\{|0\rangle+\exp \left[2 \pi i\left(0 . \xi_{n: 1} 1\right)\right]|1\rangle\right\}
\end{aligned}
$$


where $1 \leq n \leq N-1$. More precisely, the state $\left|\xi_{1: n} \cdot \xi_{n+1: N}\right\rangle$ is obtained by applying the Fourier transform operator to the $n$ rightmost bits of the position eigenstate $\left|\cdot \xi_{n+1: N} \xi_{n: 1}\right\rangle$. For given $n$, these states form an orthogonal basis. The state $\left|\xi_{1: n} \cdot \xi_{n+1: N}\right\rangle$ is localized in both position and momentum: it is strictly localized within a position region of width $1 / 2^{N-n}$, centered at position $q=0 . \xi_{n+1: N} 1$, and it is crudely localized within a momentum region of width $1 / 2^{n}$, centered at momentum $p=0 . \xi_{n: 1} 1$.

For each $n, 1 \leq n \leq N-2$, a quantum baker's map can be defined by

$$
\hat{B}\left|\xi_{1: n} \cdot \xi_{n+1: N}\right\rangle \stackrel{\text { def }}{=}\left|\xi_{1: n+1} \cdot \xi_{n+2: N}\right\rangle
$$

where the dot is shifted by one position. In phase-space language, the map $\hat{B}$ takes a state localized at $(q, p)=\left(0 . \xi_{n+1: N} 1,0 . \xi_{n: 1} 1\right)$ to a state localized at $\left(q^{\prime}, p^{\prime}\right)=\left(0 . \xi_{n+2: N} 1,0 . \xi_{n+1: 1} 1\right)$, while it stretches the state by a factor of two in the $q$ direction and squeezes it by a factor of two in the $p$ direction. This analogy with the classical baker's map motivates calling the maps (12) "quantum baker's maps." For $n=N-1$, the map is the original quantum baker's map as defined in Ref. [2], which in our notation becomes

$$
\hat{B}\left|\xi_{1: N-1} \cdot \xi_{N}\right\rangle \stackrel{\text { def }}{=}\left|\xi_{1: N} \cdot\right\rangle
$$

and for $n=0$, the map is

$$
\hat{B}\left|\cdot \xi_{1: N}\right\rangle \stackrel{\text { def }}{=}\left|\xi_{1} \cdot \xi_{2: N}\right\rangle \text {. }
$$

Below we show that all the maps $(12,13,14)$ reduce to the classical baker's map in the limit $\hbar \rightarrow 0$.

\section{Results}

Equation (12) is a mixed representation of the quantum baker's map, using different bases on both sides of the equation. To go beyond the heuristic phase-space interpretation of the map given at the end of the last section, we need to express the matrix elements of $\hat{B}$ with respect to a single basis, i.e., we need to find

$$
C^{1 \text { st }}\left(\boldsymbol{\xi}^{0}, \boldsymbol{\xi}^{1}\right) \stackrel{\text { def }}{=} \begin{cases}\left\langle\cdot \xi_{1: N}^{1}|\hat{B}| \cdot \xi_{1: N}^{0}\right\rangle & \text { if } n=0 \\ \left\langle\xi_{1: n}^{1} \cdot \xi_{n+1: N}^{1}|\hat{B}| \xi_{1: n}^{0} \cdot \xi_{n+1: N}^{0}\right\rangle & \text { if } 1 \leq n \leq N-1,\end{cases}
$$

where $\boldsymbol{\xi}^{0}=\xi_{1: N}^{0}$ and $\boldsymbol{\xi}^{1}=\xi_{1: N}^{1}$. A main result of this paper is the following simple formula, which will be proved in Sec. 田:

$$
C^{1 \mathrm{st}}\left(\boldsymbol{\xi}^{0}, \boldsymbol{\xi}^{1}\right)=\Phi\left(\xi_{1}^{0}, \xi_{N}^{1}\right) \frac{\delta\left(\xi_{n+2: N}^{0}-\xi_{n+1: N-1}^{1}\right)}{2^{n+1} \sin \left[\pi\left(0 . \xi_{n+1: 1}^{0} 1-0 . \xi_{n: 1}^{1} 1\right)\right]},
$$

where $1 \leq n \leq N-2$ and $\Phi$ is a phase factor given by

$$
\Phi\left(\xi_{1}^{0}, \xi_{N}^{1}\right)=\frac{1}{\sqrt{2}}\left[i(-1)^{\xi_{N}^{1}}-(-1)^{\xi_{1}^{0}}\right]
$$

For the case $n=0$, one obtains

$$
C^{1 \mathrm{st}}\left(\boldsymbol{\xi}^{0}, \boldsymbol{\xi}^{1}\right)=\frac{1-i}{2} \delta\left(\xi_{2: N}^{0}-\xi_{1: N-1}^{1}\right) e^{i \frac{\pi}{2}\left|\xi_{1}^{0}-\xi_{N}^{1}\right|},
$$


and for $n=N-1$,

$$
C^{1 \mathrm{st}}\left(\boldsymbol{\xi}^{0}, \boldsymbol{\xi}^{1}\right)=\frac{\Phi\left(\xi_{1}^{0}, \xi_{N}^{1}\right)}{2^{N} \sin \left[\pi\left(0 . \xi_{N: 1}^{0} 1-0 . \xi_{N-1: 1}^{1} 1\right)\right]}
$$

The coefficients $C^{1 s t}\left(\boldsymbol{\xi}^{0}, \boldsymbol{\xi}^{1}\right)$ given in 16$)$ are zero unless $\xi_{n+2: N}^{0}=\xi_{n+1: N-1}^{1}$, i.e., unless the position bits $\xi_{n+1: N-1}^{1}$ of the final state are obtained by shifting the corresponding position bits of the initial state. Furthermore, the sin term in the denominator ensures that $C^{1 \text { st }}$ is strongly peaked for $\xi_{n+1: 2}^{0}=\xi_{n: 1}^{1}$, i.e., if the momentum bits $\xi_{n: 1}^{1}$ of the final state are obtained by shifting the corresponding momentum bits of the initial state. The formula (16) therefore establishes rigorously that the maps (12) are approximate shift maps, a result which had been obtained numerically in Ref. [5].

To formulate the question of the classical limit of the baker's map, we use the concept of coarsegraining in the spirit of the consistent (or decoherent) histories approach [22, 23, 24]. For this, we introduce projectors on subspaces corresponding to symbolic strings $\boldsymbol{y}$ of length $l$. We fix in advance an upper limit, $k_{\max }$, on the number of iterations, $k$, considered; this is necessary because in computing the classical limit of a chaotic map, the limit $\hbar=2^{-(N+1)} / \pi \rightarrow 0$ has to be taken before the limit $k \rightarrow \infty$ [25]. We will show that, for given $l$ and $k_{\max }$, it is always possible to choose $\hbar$ in such a way that the coarse-grained quantum dynamics is arbitrarily close to a shift of the string $\boldsymbol{y}$. In contrast to the approach of Refs. [18, 19], in taking the limit $\hbar \rightarrow 0$, we always remain in the finite-dimensional Hilbert space on which our maps are defined.

As before, we are considering basis states of the form $\left|\xi_{1: n} . \xi_{n+1: N}\right\rangle$. As we let $N$ increase, the number of position bits to the right of the dot, $m \stackrel{\text { def }}{=} N-n$, remains fixed. We define $r=N-l$ as the number of bits ignored in the coarse graining. In the following, we always assume that $k \leq k_{\max }<r$.

We are now in a position to introduce a family of projectors

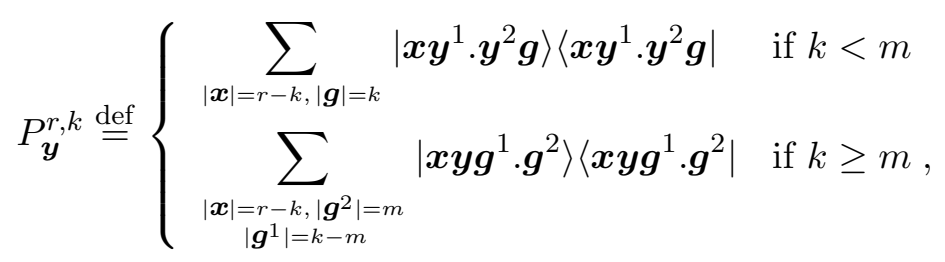

where $\boldsymbol{y}^{1} \boldsymbol{y}^{2}=\boldsymbol{y}$ and $\left|\boldsymbol{y}^{2}\right|=m-k$. By normalizing these projectors, we obtain a family of uniform density matrices,

$$
\rho_{k} \stackrel{\text { def }}{=} 2^{-r} P_{\boldsymbol{y}}^{r, k}
$$

A classical shift acts on these states as

$$
\rho_{k} \mapsto \rho_{k+1}
$$

Projecting a state $\rho_{k^{\prime}}$ onto the shifted subspace $P_{\boldsymbol{y}}^{r, k}$ gives the characteristic delta distribution

$$
\operatorname{Tr}\left[P_{\boldsymbol{y}}^{r, k} \rho_{k^{\prime}}\right]=\delta_{k k^{\prime}} .
$$

We will prove that

$$
\operatorname{Tr}\left[P_{\boldsymbol{y}}^{r, k} \hat{B}^{k} \rho_{0}\left(\hat{B}^{\dagger}\right)^{k}\right]=1-O\left(\frac{r}{2^{r-k}}\right)
$$


or, since $k$ is bounded from above by $k_{\max }$, and $r=N-l$, where $l$ is fixed,

$$
\operatorname{Tr}\left[P_{\boldsymbol{y}}^{r, k} \hat{B}^{k} \rho_{0}\left(\hat{B}^{\dagger}\right)^{k}\right]=1-O\left(\frac{N}{2^{N}}\right)=1-O(\hbar \log \hbar) .
$$

Comparing Eqs. (23) and (25), one sees that the coarse-grained quantum evolution approaches the

shift-map behavior to any required accuracy as $\hbar \rightarrow 0$. A measurement of the projectors $P_{\boldsymbol{y}}^{r, k}$ can be interpreted as a measurement in which the $r-k$ leftmost bits and the $k$ rightmost bits of the symbolic string are not resolved.

Equation (24) can be rewritten as

$$
2^{-r} \sum_{|\boldsymbol{x}|=r} \operatorname{Tr}\left[P_{\boldsymbol{y}}^{r, k} \hat{B}^{k}\left|\boldsymbol{x} \boldsymbol{y}^{1} \cdot \boldsymbol{y}^{2}\right\rangle\left\langle\boldsymbol{x} \boldsymbol{y}^{1} \cdot \boldsymbol{y}^{2}\right|\left(\hat{B}^{\dagger}\right)^{k}\right]=1-O\left(\frac{r}{2^{r-k}}\right),
$$

which is a sum of $2^{r}$ terms bounded from above as

$$
\operatorname{Tr}\left[P_{\boldsymbol{y}}^{r, k} \hat{B}^{k}\left|\boldsymbol{x} \boldsymbol{y}^{1} \cdot \boldsymbol{y}^{2}\right\rangle\left\langle\boldsymbol{x} \boldsymbol{y}^{1} \cdot \boldsymbol{y}^{2}\right|\left(\hat{B}^{\dagger}\right)^{k}\right] \leq 1
$$

Here, $\boldsymbol{y}^{1} \boldsymbol{y}^{2}=\boldsymbol{y}$ and $\left|\boldsymbol{y}^{2}\right|=m$ as before. Equations (26) and (27) can be both satisfied only if the condition

$$
\operatorname{Tr}\left[P_{\boldsymbol{y}}^{r, k} \hat{B}^{k}\left|\boldsymbol{x} \boldsymbol{y}^{1} \cdot \boldsymbol{y}^{2}\right\rangle\left\langle\boldsymbol{x} \boldsymbol{y}^{1} \cdot \boldsymbol{y}^{2}\right|\left(\hat{B}^{\dagger}\right)^{k}\right]=1-O\left(\frac{r}{2^{r-k}}\right)
$$

holds for all $\boldsymbol{x}$ except for a fraction of order $r / 2^{r-k}$, i.e., for all basis states $\left|\xi_{1: n} \cdot \xi_{n+1: N}\right\rangle$ except for an exponentially small fraction. In other words, the property (28) holds for typical basis states.

An interesting feature of the quantum baker's map is that there are atypical basis states for which Eq. (28) does not hold. In section IV we give an example of an atypical state $\left|\boldsymbol{x}^{\text {atyp }} \boldsymbol{y}^{1} \cdot \boldsymbol{y}^{2}\right\rangle$ for which

$$
\operatorname{Tr}\left[P_{\boldsymbol{y}}^{r, 1} \hat{B}\left|\boldsymbol{x}^{\text {atyp }} \boldsymbol{y}^{1} \cdot \boldsymbol{y}^{2}\right\rangle\left\langle\boldsymbol{x}^{\text {atyp }} \boldsymbol{y}^{1} \cdot \boldsymbol{y}^{2}\right| \hat{B}^{\dagger}\right]=\frac{\pi^{2}+8 G}{2 \pi^{2}}+O\left(4^{r-n}\right)+O\left(2^{-r}\right),
$$

where $G \simeq 0.915965$ is Catalan's constant [26]. For sufficiently large $n-r$ and $r$, this expression is less than 0.872. This is an example where the quantum evolution in the limit $\hbar \rightarrow 0$ differs substantially from the classical evolution, already after the first iteration of the map. If, however, the initial state is a mixture in which atypical states have an exponentially small weight, such as $\rho_{0}$, the correspondence principle is obeyed.

\section{Derivations and proofs}

\subsection{First Iteration}

In this section we prove the formula (16) for the matrix elements $C^{1 \mathrm{st}}\left(\boldsymbol{\xi}^{0}, \boldsymbol{\xi}^{1}\right)$. Equation (19) for the case $n=N-1$ follows from almost identical arguments, and Eq. (18) for $n=0$ is essentially trivial. A direct calculation yields

$$
\begin{aligned}
C^{1 \mathrm{st}}\left(\boldsymbol{\xi}^{0}, \boldsymbol{\xi}^{1}\right)= & \delta\left(\xi_{n+2}^{0}-\xi_{n+1}^{1}\right) \delta\left(\xi_{n+3}^{0}-\xi_{n+2}^{1}\right) \cdots \delta\left(\xi_{N}^{0}-\xi_{N-1}^{1}\right) \\
& \times \sqrt{1 / 2}\left\{\delta\left(\xi_{N}^{0}\right)+\delta\left(\xi_{N}^{0}-1\right) \exp \left[2 \pi i\left(0 . \xi_{1}^{0} 1\right)\right]\right\} \\
& \times \exp \left\{i \pi\left(0 . \xi_{n+1: 1}^{0} 1-0 . \xi_{n: 1}^{1} 1\right)\right\}
\end{aligned}
$$




$$
\begin{aligned}
& \times 1 / 2\left[1+\exp \left\{2 \pi i\left(0 . \xi_{2}^{0} \xi_{1}^{0} 1-0 . \xi_{1}^{1} 1\right)\right\}\right] \times \cdots \\
& \times 1 / 2\left[1+\exp \left\{2 \pi i\left(0 . \xi_{n+1: 1}^{0} 1-0 . \xi_{n: 1}^{1} 1\right)\right\}\right]
\end{aligned}
$$

Using the identity $1+e^{i \phi}=2 e^{i \phi / 2} \cos (\phi / 2)$ and noticing that $\delta\left(\xi_{N}^{1}\right)+\delta\left(\xi_{N}^{1}-1\right) \exp \left[2 \pi i\left(0 . \xi_{1}^{0} 1\right)\right]$ $=\exp \left[i \pi \xi_{N}^{1}\left(\xi_{1}^{0}+1 / 2\right)\right]$, we have

$$
\begin{aligned}
C^{1 \mathrm{st}}\left(\boldsymbol{\xi}^{0}, \boldsymbol{\xi}^{1}\right)= & \sqrt{1 / 2} \exp \left[i \pi \xi_{N}^{1}\left(\xi_{1}^{0}+1 / 2\right)\right] \delta\left(\xi_{n+2: N}^{0}-\xi_{n+1: N-1}^{1}\right) \\
& \times \exp \left[i \pi\left(0 . \xi_{n+1: 1}^{0} 1-0 . \xi_{n: 1}^{1} 1\right)\right] \\
& \times \prod_{k=2}^{n+1} \cos \left[\pi\left(0 . \xi_{k: 1}^{0} 1-0 . \xi_{k-1: 1}^{1} 1\right)\right] \\
& \times \prod_{k=2}^{n+1} \exp \left[i \pi\left(0 . \xi_{k: 1}^{0} 1-0 . \xi_{k-1: 1}^{1} 1\right)\right] \\
= & \sqrt{1 / 2} \exp \left[i \pi \xi_{N}^{1}\left(\xi_{1}^{0}+1 / 2\right)\right] \delta\left(\xi_{n+2: N}^{0}-\xi_{n+1: N-1}^{1}\right) \\
& \times e^{i \phi_{n}}\left(\prod_{k=1}^{n} \cos \phi_{k}\right)\left(\prod_{k=1}^{n} e^{i \phi_{k}}\right)
\end{aligned}
$$

where

$$
\phi_{k} \stackrel{\text { def }}{=} \pi\left(0 . \xi_{k+1: 1}^{0} 1-0 . \xi_{k: 1}^{1} 1\right) \text {. }
$$

To simplify Eq. (31), we first consider the products of cosines and exponents separately and then combine them to formulate the final result for the first iteration of the baker's map. Note that

$$
\begin{aligned}
2 \phi_{k} & =\pi\left(0 . \xi_{k: 1}^{0} 1-0 . \xi_{k-1: 1}^{1} 1\right)+\pi\left(\xi_{k+1}^{0}-\xi_{k}^{1}\right) \\
& =\phi_{k-1}+\pi\left(\xi_{k+1}^{0}-\xi_{k}^{1}\right)
\end{aligned}
$$

so

$$
\begin{aligned}
\cos \phi_{k-1} & =\cos \left[2 \phi_{k}+\pi\left(\xi_{k}^{1}-\xi_{k+1}^{0}\right)\right] \\
& =(-1)^{\xi_{k}^{1}-\xi_{k+1}^{0}} \cos \left(2 \phi_{k}\right), \quad k \leq n .
\end{aligned}
$$

From Eq. (33), we have $2 \phi_{k}=4 \phi_{k+1}(\bmod 2 \pi)$ and thus $2 \phi_{k}=2^{n+1-k} \phi_{n}(\bmod 2 \pi)$, so the previous formula can be rewritten as

$$
\cos \phi_{k}=(-1)^{\xi_{k+1}^{1}-\xi_{k+2}^{0} \cos \left(2^{n-k} \phi_{n}\right), \quad k \leq n-1 .}
$$

Using this formula the product of cosines can be expressed as

$$
\begin{aligned}
\prod_{k=1}^{n} \cos \phi_{k} & =\cos \phi_{n} \prod_{k=1}^{n-1} \cos \phi_{k} \\
& =(-1)^{\sigma\left(\xi_{2: n}^{1}\right)-\sigma\left(\xi_{3: n+1}^{0}\right)} \prod_{k=1}^{n} \cos \left[2^{-k}\left(2^{n} \phi_{n}\right)\right]
\end{aligned}
$$

where $\sigma\left(\xi_{k: n}\right) \stackrel{\text { def }}{=} \sum_{s=k}^{n} \xi_{s}$. It is easy to check by induction that

$$
\prod_{k=0}^{n-1} \cos 2^{k} x=\frac{\sin 2^{n} x}{2^{n} \sin x}, \quad x \neq \pi j, \quad j=0, \pm 1, \pm 2, \ldots
$$


In our case

$$
\prod_{k=1}^{n} \cos \left[2^{-k}\left(2^{n} \phi_{n}\right)\right]=\frac{\sin \left(2^{n} \phi_{n}\right)}{2^{n} \sin \phi_{n}}
$$

Putting everything together, the product of cosines becomes

$$
\prod_{k=1}^{n} \cos \phi_{k}=(-1)^{\sigma\left(\xi_{2: n}^{1}\right)-\sigma\left(\xi_{3: n+1}^{0}\right)} \frac{\sin \left(2^{n} \phi_{n}\right)}{2^{n} \sin \phi_{n}}
$$

where $\phi_{n}=\pi\left(0 . \xi_{n+1: 1}^{0} 1-0 . \xi_{n: 1}^{1} 1\right)$. Now we simplify the product of exponents in (31). Equation (33) implies

$$
\phi_{n-k}=2^{k} \phi_{n}+\sum_{s=1}^{k} 2^{k-s} \pi\left(\xi_{n+1-s}^{1}-\xi_{n+2-s}^{0}\right), k \geq 1
$$

so

$$
\begin{aligned}
\sum_{k=1}^{n} \phi_{k} & =\phi_{n}+\sum_{k=1}^{n-1} \phi_{n-k} \\
& =\phi_{n} \sum_{k=0}^{n-1} 2^{k}+\sum_{k=1}^{n-1} \sum_{s=1}^{k} 2^{k-s} \pi\left(\xi_{n+1-s}^{1}-\xi_{n+2-s}^{0}\right) \\
& =\left[\phi_{n}\left(2^{n}-1\right)+\sum_{k=1}^{n-1} \pi\left(\xi_{n+1-k}^{1}-\xi_{n+2-k}^{0}\right)\right](\bmod 2 \pi) \\
& =\left\{\phi_{n}\left(2^{n}-1\right)+\pi\left[\sigma\left(\xi_{2: n}^{1}\right)-\sigma\left(\xi_{3: n+1}^{0}\right)\right]\right\}(\bmod 2 \pi) .
\end{aligned}
$$

The product of exponents is thus given by

$$
\prod_{k=1}^{n} e^{i \phi_{k}}=\exp \left(i \sum_{k=1}^{n} \phi_{k}\right)=(-1)^{\sigma\left(\xi_{2: n}^{1}\right)-\sigma\left(\xi_{3: n+1}^{0}\right)} \frac{\exp \left(i 2^{n} \phi_{n}\right)}{\exp \left(i \phi_{n}\right)} .
$$

Using (39) and (42) one can rewrite (31) as

$$
C^{1 \mathrm{st}}\left(\boldsymbol{\xi}^{0}, \boldsymbol{\xi}^{1}\right)=\frac{(-1)^{\xi_{N}^{1}\left(\xi_{1}^{0}+1 / 2\right)}}{2^{n+1 / 2}} \delta\left(\xi_{n+2: N}^{0}-\xi_{n+1: N-1}^{1}\right) \frac{\sin \left(2^{n} \phi_{n}\right)}{\sin \phi_{n}} \exp \left(i 2^{n} \phi_{n}\right) .
$$

Further simplification is possible due to the fact that $2^{n} \phi_{n}=2 \pi\left(0 . \xi_{2}^{0} \xi_{1}^{0} 1-0 . \xi_{1}^{1} 1\right)(\bmod 2 \pi)$. The final result is

$$
C^{1 \mathrm{st}}\left(\boldsymbol{\xi}^{0}, \boldsymbol{\xi}^{1}\right)=\Phi\left(\xi_{1}^{0}, \xi_{N}^{1}\right) \frac{\delta\left(\xi_{n+2: N}^{0}-\xi_{n+1: N-1}^{1}\right)}{2^{n+1} \sin \left[\pi\left(0 . \xi_{n+1: 1}^{0} 1-0 . \xi_{n: 1}^{1} 1\right)\right]},
$$

where the phase factor $\Phi$ is given by

$$
\begin{aligned}
\Phi\left(\xi_{1}^{0}, \xi_{N}^{1}\right) & =\sqrt{2} e^{i \pi \xi_{N}^{1}\left(\xi_{1}^{0}+1 / 2\right)} \sin \left(2^{n} \phi_{n}\right) \exp \left(i 2^{n} \phi_{n}\right) \\
& =\frac{1}{\sqrt{2}}\left[i(-1)^{\xi_{N}^{1}}-(-1)^{\xi_{1}^{0}}\right]
\end{aligned}
$$

This formula is an exact expression for the matrix elements (15) of the quantum baker's map. 


\section{$4.2 \quad k$-th iteration}

In this section we prove that for all $k \leq k_{\max }$,

$$
\operatorname{Tr}\left[P_{\boldsymbol{y}}^{r, k} \hat{B}^{k} \rho_{0}\left(\hat{B}^{\dagger}\right)^{k}\right]=1-O\left(\frac{r}{2^{r-k}}\right),
$$

where the projectors $P_{\boldsymbol{y}}^{r, k}$ and the density operators $\rho_{j}$ are defined in Eqs. (20) and (21). The first step is to prove that

$$
\operatorname{Tr}\left[P_{\boldsymbol{y}}^{r, k} \hat{B} \rho_{k-1} \hat{B}^{\dagger}\right]=1-O\left(\frac{r}{2^{r-k}}\right) .
$$

By a direct calculation, we obtain

$$
\begin{aligned}
\operatorname{Tr}\left[P_{\boldsymbol{y}}^{r, k} \hat{B} \rho_{k-1} \hat{B}^{\dagger}\right] & =2^{k-r} \sum_{\substack{|\boldsymbol{\alpha}|=r-k+1 \\
|\boldsymbol{\beta}|=r-k}}\left|2^{n+1} \sin \left[2^{-(n-r+k)} \pi(0 . \boldsymbol{\alpha} 1-0 . \boldsymbol{\beta} 1)\right]\right|^{-2} \\
& \geq \frac{4}{\pi^{2} 2^{r-k}} \sum_{u=0}^{2^{r-k+1}} \sum_{v=0}^{2^{r-k}-1}(2 u-4 v-1)^{-2}
\end{aligned}
$$

where the inequality $\sin ^{2} x \leq x^{2}$ was used. Let $L=r-k$, and let $Q(s)$ be the number of different pairs $(u, v), 0 \leq u<2^{L+1}, 0 \leq v<2^{L}$, for which $u-2 v=s$. It follows that

$$
\begin{aligned}
\sum_{u=0}^{2^{r-k+1}-1} \sum_{v=0}^{2^{r-k}-1}(2 u-4 v-1)^{-2} & =\sum_{s=-2\left(2^{L}-1\right)}^{2^{L+1}-1} Q(s)(2 s-1)^{-2} \\
& =\sum_{s=1}^{2^{L+1}-1} \frac{Q(s)+Q(1-s)}{(2 s-1)^{2}}
\end{aligned}
$$

Using a simple counting argument based on the register principle [28], one can show that

$$
Q(s)+Q(1-s)=2^{L+1}-s+\frac{1}{2}\left[1-(-1)^{s}\right],
$$

from which one obtains

$$
\begin{aligned}
\sum_{s=1}^{2^{L+1}-1} \frac{Q(s)+Q(1-s)}{(2 s-1)^{2}} & =\sum_{s=1}^{2^{L}-1} \frac{2^{L+1}-s}{(2 s-1)^{2}}-\sum_{t=1}^{2^{L}-1}(4 t-1)^{-2} \\
& =2^{L}\left[\frac{\pi^{2}}{4}-O\left(\frac{L}{2^{L}}\right)\right]
\end{aligned}
$$

where we have used the relations

$$
\sum_{s=1}^{2^{L}}(2 s-1)^{-2}=\frac{\pi^{2}}{8}+O\left(2^{-L}\right), \quad 2^{-L} \sum_{s=1}^{2^{L}} \frac{1}{2 s-1}=O\left(\frac{L}{2^{L}}\right) .
$$

Combining Eqs. (48), (49) and (51), we obtain Eq. (47) as required. We now rewrite Eq. (47) in the symmetric form

$$
\operatorname{Tr}\left[\rho_{k} \hat{B} \rho_{k-1} \hat{B}^{\dagger}\right]=2^{-r}\left[1-O\left(\frac{r}{2^{r-k}}\right)\right]
$$


and introduce the distance measure between density matrices induced by the Eucledian norm [27],

$$
d\left(\rho, \rho^{\prime}\right) \stackrel{\text { def }}{=} \sqrt{\operatorname{Tr}\left(\rho-\rho^{\prime}\right)^{2}} .
$$

This distance measure is unitarily invariant and obeys the triangle inequality. We will now prove that (53) and (54) imply

$$
d\left(\rho_{k}, \hat{B}^{k} \rho_{0}\left[\hat{B}^{\dagger}\right]^{k}\right)=O\left(2^{\frac{k}{2}-r} \sqrt{r}\right)
$$

Using the cyclic property of the trace, we have

$$
d\left(\rho_{k}, \hat{B} \rho_{k-1} \hat{B}^{\dagger}\right)=\sqrt{\operatorname{Tr} \rho_{k}^{2}+\operatorname{Tr} \rho_{k-1}^{2}-2 \operatorname{Tr}\left(\rho_{k} \hat{B} \rho_{k-1} \hat{B}^{\dagger}\right)} .
$$

Since $\operatorname{Tr} \rho_{k}^{2}=2^{r} / 2^{2 r}=2^{-r}$ for any $k$,

$$
\left[d\left(\rho_{k}, \hat{B} \rho_{k-1} \hat{B}^{\dagger}\right)\right]^{2}=2^{-r+1}-2 \operatorname{Tr}\left(\rho_{k} \hat{B} \rho_{k-1} \hat{B}^{\dagger}\right),
$$

which, together with Eq. (53), implies

$$
d\left(\rho_{k}, \hat{B} \rho_{k-1} \hat{B}^{\dagger}\right)=O\left(2^{\frac{k}{2}-r} \sqrt{r}\right) .
$$

The case $k=1$ of (55) follows directly from (58). Assuming that (55) is true for a given value of $k$ and using the unitary invariance of the distance (54), we have

$$
d\left(\hat{B} \rho_{k} \hat{B}^{\dagger}, \hat{B}^{k+1} \rho_{0}\left[\hat{B}^{\dagger}\right]^{k+1}\right)=O\left(2^{\frac{k}{2}-r} \sqrt{r}\right) .
$$

Substituting $k+1$ for $k$ in Eq. (58), we get

$$
d\left(\rho_{k+1}, \hat{B} \rho_{k} \hat{B}^{\dagger}\right)=O\left(2^{\frac{1}{2}(k+1)-r} \sqrt{r}\right) .
$$

Using the triangle inequality for the distance measure (54), it follows from (59) and (60) that

$$
\begin{aligned}
d\left(\rho_{k+1}, \hat{B}^{k+1} \rho_{0}\left[\hat{B}^{\dagger}\right]^{k+1}\right) & =O\left(2^{\frac{k}{2}-r} \sqrt{r}\right)+O\left(2^{\frac{1}{2}(k+1)-r} \sqrt{r}\right) \\
& =O\left(2^{\frac{1}{2}(k+1)-r} \sqrt{r}\right) .
\end{aligned}
$$

By induction, this completes the proof of (55) for any $k \leq k_{\max }$. On the other hand

$$
d\left(\rho_{k}, \hat{B}^{k} \rho_{0}\left[\hat{B}^{\dagger}\right]^{k}\right)=\sqrt{\operatorname{Tr} \rho_{k}^{2}+\operatorname{Tr} \rho_{0}^{2}-2 \operatorname{Tr}\left(\rho_{k} \hat{B}^{k} \rho_{0}\left[\hat{B}^{\dagger}\right]^{k}\right)},
$$

hence using Eq. (55) it follows that

$$
\sqrt{2^{1-r}-2 \operatorname{Tr}\left(\rho_{k} \hat{B}^{k} \rho_{0}\left[\hat{B}^{\dagger}\right]^{k}\right)}=O\left(2^{\frac{k}{2}-r} \sqrt{r}\right)
$$

and finally

$$
\operatorname{Tr}\left(\rho_{k} \hat{B}^{k} \rho_{0}\left[\hat{B}^{\dagger}\right]^{k}\right)=2^{-r}\left[1-O\left(\frac{r}{2^{r-k}}\right)\right],
$$

which is equivalent to (46) as required. 


\subsection{Atypical initial states}

In this section, we show that the state $\left|0^{r} \boldsymbol{y}^{1} \cdot \boldsymbol{y}^{2}\right\rangle$, where $0^{r}$ is a string of $r$ zeros, is an atypical state in the sense of the discussion at the end of Sec. 3, i.e., we show that the state $\left|0^{r} \boldsymbol{y}^{1} \cdot \boldsymbol{y}^{2}\right\rangle$ satisfies Eq. (29). A direct calculation gives

$$
\begin{aligned}
\operatorname{Tr}\left[P_{\boldsymbol{y}}^{r, 1} \hat{B}\left|0^{r} \boldsymbol{y}^{1} \cdot \boldsymbol{y}^{2}\right\rangle\left\langle 0^{r} \boldsymbol{y}^{1} \cdot \boldsymbol{y}^{2}\right| \hat{B}^{\dagger}\right] & =\sum_{|\boldsymbol{x}|=r-1} \sum_{g=0}^{1}\left|C^{1 \mathrm{st}}\left(0^{r} \boldsymbol{y}^{1} \boldsymbol{y}^{2}, \boldsymbol{x} \boldsymbol{y}^{1} \boldsymbol{y}^{2} g\right)\right|^{2} \\
& =\frac{8}{\pi^{2}} \sum_{v=0}^{2^{r-1}-1} \frac{1+O\left(4^{r-n}\right)}{(4 v+1)^{2}}
\end{aligned}
$$

Substituting $t=2 v$, we have

$$
\begin{aligned}
\operatorname{Tr}\left[P_{\boldsymbol{y}}^{r, 1} \hat{B}\left|0^{r} \boldsymbol{y}^{1} \cdot \boldsymbol{y}^{2}\right\rangle\left\langle 0^{r} \boldsymbol{y}^{1} \cdot \boldsymbol{y}^{2}\right| \hat{B}^{\dagger}\right] & =\frac{8+O\left(4^{r-n}\right)}{\pi^{2}} \sum_{t=0}^{2^{r}} \frac{1+(-1)^{t}}{2(2 t+1)^{2}} \\
& =\frac{4+O\left(4^{r-n}\right)}{\pi^{2}}\left(\sum_{s=1}^{2^{r}+1}(2 s-1)^{-2}+\sum_{t=0}^{2^{r}} \frac{(-1)^{t}}{(2 t+1)^{2}}\right) .
\end{aligned}
$$

Using Eq. (52) and the series representation of Catalan's constant $G \simeq 0.915965$ 26],

$$
G=\sum_{t=0}^{\infty} \frac{(-1)^{t}}{(2 t+1)^{2}},
$$

it follows that

$$
\begin{aligned}
\operatorname{Tr}\left[P_{\boldsymbol{y}}^{r, 1} \hat{B}\left|0^{r} \boldsymbol{y}^{1} \cdot \boldsymbol{y}^{2}\right\rangle\left\langle 0^{r} \boldsymbol{y}^{1} \cdot \boldsymbol{y}^{2}\right| \hat{B}^{\dagger}\right] & =\frac{\pi^{2}+8 G}{2 \pi^{2}}+O\left(4^{r-n}\right)+O\left(2^{-r}\right) \\
& \simeq 0.871+O\left(4^{r-n}\right)+O\left(2^{-r}\right)
\end{aligned}
$$

Since one can treat $n-r$ and $r$ as independent variables, this expression can be made smaller than 0.872 by choosing $n-r$ and $r$ large enough. For the initial state $\left|0^{r} \boldsymbol{y}^{1} \cdot \boldsymbol{y}^{2}\right\rangle$, the asymptotic relation (28) is thus violated.

\section{Acknowledgements}

Thanks to C. M. Caves, C. A. Fuchs and M. Saraceno for useful discussions. We acknowledge the hospitality of the Isaac Newton Institute in Cambridge, where part of this work was completed. This work was supported in part by the US Office of Naval Research (Grant No. N00014-93-0-0116) and by the UK Engineering and Physical Sciences Research Council.

\section{References}

[1] N. L. Balazs and A. Voros, Ann. Phys. 190, 1 (1989).

[2] M. Saraceno, Ann. Phys. 199, 37 (1990).

[3] A. M. Ozorio de Almeida and M. Saraceno, Ann. Phys. 210, 1 (1991). 
[4] F. M. Dittes, E. Doron, and U. Smilansky, Phys. Rev. E 49, R963 (1994).

[5] M. Saraceno and A. Voros, Physica D 79, 206 (1994).

[6] A. Lakshminarayan, Ann. Phys. 239, 272 (1995).

[7] L. Kaplan and E. J. Heller, Phys. Rev. Lett. 76, 1453 (1996).

[8] M. G. E. da Luz and A. M. Ozorio de Almeida, Nonlinearity 8, 43 (1995).

[9] R. Schack and C. M. Caves, Phys. Rev. Lett. 71, 525 (1993).

[10] R. Schack and C. M. Caves, Phys. Rev. E 53, 3257 (1996).

[11] J. H. Hannay, J. P. Keating, and A. M. Ozorio de Almeida, Nonlinearity 7, 1327 (1994).

[12] R. Schack, Phys. Rev. A 57, 1634 (1998).

[13] T. A. Brun and R. Schack, Phys. Rev. A 59, 2649 (1999).

[14] P. Pakonski, A. Ostruszka, and K. Życzkowski, Nonlinearity 12, 269 (1999).

[15] V. I. Arnold and A. Avez, Ergodic Problems of Classical Mechanics (Benjamin, New York, 1968).

[16] M. V. Berry, N. L. Balazs, M. Tabor, and A. Voros, Ann. Phys. 122, 26 (1979).

[17] H. Weyl, The Theory of Groups and Quantum Mechanics (Dover, New York, 1950).

[18] R. Rubin and N. Salwen, Ann. Phys. 269, 159 (1998).

[19] A. Lesniewski, R. Rubin, and N. Salwen, J. Math. Phys. 39, 1835 (1998).

[20] R. Schack and C. M. Caves, to appear in AAECC, e-print quant-ph/9810050.

[21] V. M. Alekseev and M. V. Yakobson, Phys. Reports 75, 287 (1981).

[22] R. Griffiths, J. Stat. Phys. 36, 219 (1984).

[23] R. Omnès, J. Stat. Phys. 53, 893, 933, 957 (1988).

[24] M. Gell-Mann and J. B. Hartle, Phys. Rev. D 47, 3345 (1993).

[25] M. V. Berry, in Chaos and Quantum Physics, edited by M.-J. Giannoni, A. Voros, and J. Zinn-Justin (North-Holland, Amsterdam, 1991).

[26] I. S. Gradshteyn and I. M. Ryzhik, Table of Integrals, Series, and Products (Academic Press, New York, 1994).

[27] R. A. Horn and C. R. Johnson, Matrix Analysis (Cambridge University Press, Cambridge, 1996).

[28] H. F. Mattson, Jr., Discrete Mathematics with Applications (Wiley, New York, 1993). 